\title{
Eficiência técnica da Polícia Militar em Minas Gerais
}

Paulo Roberto Scalco Professor da Faculdade de Administraçăo, Ciências Contábeis e Economia da Universidade Federal de Goiás (FACE-UFG).

Airton Lopes Amorim

Economista M.Sc. e doutorando em Economia Aplicada pela Universidade Federal de Viçosa (UFV). Adriano Provezano Gomes Professor do Departamento de Economia da Universidade Federal de Viçosa (UFV).

\section{Palauras-chave \\ criminalidade, segurança pública, políticas públicas, Análise Envoltória de Dados (DEA).}

Classificação JEL C60, H76, R58.

\footnotetext{
Key words

criminality, public safety, public policies, Data Envelopment Analysis (DEA)

JEL Classification $\mathrm{C} 60$, $H 76$, R58
}

\section{Resumo}

Calcular um índice de eficiência no combate à criminalidade, que permitisse a comparação entre as unidades da Polícia Militar no Estado de Minas Gerais, foi o objetivo deste trabalho. Os índices de eficiência foram calculados por meio da técnica Data Envelopment Analysis e os resultados apontaram para uma grande discrepância entre os municípios analisados de tal forma que uma sugestão de política de segurança pública seria buscar a elevação dos índices de eficiência nos municípios com desempenho pouco satisfatório, utilizando aqueles mais eficientes como referência. Além disso, um modelo Tobit também foi estimado para testar a relevância que o ambiente socioeconômico exercia na obtenção dos índices de eficiência. Os resultados mostraram que o conjunto de variáveis utilizadas explicou apenas $10 \%$ da variabilidade dos índices calculados.

\section{Abstract}

This study sought to calculate an efficiency index in crime fighting that would allow comparison between Minas Gerais State military police units. The efficiency index were calculated by Data Envelopment Analysis and the results indicated a large discrepancy between the municipalities analyzed and possible public security policy could try to raise efficiency levels in the worst municipalities using the most efficient ones as benchmarks. Furthermore, a Tobit model was estimated to test the relevance of the social and economic environmental exercised in achieving the efficiency index. The results showed that the set of variables used explained only $10 \%$ of the variability of the index calculated. 


\section{1_Introdução}

Ao longo das últimas décadas, o tema segurança pública vem sendo cada vez mais discutido tanto no Brasil quanto em outras partes do mundo. $\mathrm{O}$ expressivo crescimento das taxas de criminalidade, associado à brutalidade com que muitos crimes são cometidos, justifica a crescente preocupação quanto a esse assunto.

Muito se tem discutido a respeito de políticas de segurança pública que visem a reduzir as taxas de criminalidade; no entanto, pouco dessa discussão tem evoluído em termos de políticas públicas eficazes, e, na maioria das vezes, as políticas existentes são constituídas sob critérios de senso comum, sem quaisquer ideias ou práticas de monitoramento e avaliação (Lima et al., 2000 apud Batitucci et al., 2003).

Uma dificuldade adicional na definiçãao de políticas eficazes de combate ao crime se deve à própria complexidade do assunto. Para Beato Filho (1999), por exemplo, um dos obstáculos para identificar as variáveis responsáveis pelo crime está no fato de esse tratar de um conceito que envolve comportamentos diferentes, bem como enorme diversidade de eventos, que vão desde roubar uma revista em quadrinhos ou sonegar impostos até roubar um banco ou assassinar alguém. A heterogeneidade desses eventos gera dificuldades para a formulação de políticas públicas, já que significa identificar fatores de risco distintos a cada situação. Por outro lado, um ponto que parece consensual nesse debate é que muitas das políticas necessárias à redução dos índices de criminalidade são de natureza estrutural, cuja implementação haveria de se dar em longo prazo.

No entanto, a sensação de insegurança vivenciada pela sociedade cria uma demanda por medidas de curto prazo, que visem, se não a conter, ao menos a retardar o crescimento das taxas de crimes violentos. Neste sentido, a eficiência dos agentes envolvidos no combate à criminalidade torna-se um assunto de pesquisa que pode ser explorado, ou seja, a identificação de fatores que geram ineficiências em determinados setores seria uma forma importante de definir ações que melhorariam o combate à criminalidade. ${ }^{1}$

Nessa perspectiva, o objetivo inicial deste trabalho foi calcular um índice de eficiência técnica da Polícia Militar de Minas Gerais no combate à criminalidade. Os escores de eficiência técnica foram calculados para os municípios do Estado, com base na técnica de Data Envelopment Analysis (DEA). Posteriormente, procurou-se testar algumas hipóteses sobre os determinantes dos índices de
1 Questôes relacionadas à eficiência são, frequentemente, abordadas por pesquisadores e tomadores de decisão, principalmente em se tratando de ambientes competitivos e dinâmicos. Identificar o verdadeiro potencial de expansão da produçáo e as mudanças na eficiência, no progresso tecnológico e na produtividade ao longo do tempo, é condição necessária para formulação de políticas econômicas coerentes com as reais necessidades dos setores analisados. Por isso, o uso de medidas de eficiência tem crescido significativamente nas últimas décadas, tornando-se, hoje em dia, um dos principais tópicos de estudo dos economistas (Gomes; Baptista, 2004). 
2 Neste ponto, não se está se referindo à presença física da polícia, mas sim à percepçáo junto à sociedade da instituiçáo como autoridade e responsável pela manutenção da segurança pública. eficiência calculados. No entanto, como destacado na seção três, em razão das limitaçôes impostas pela falta de dados disponíveis, limitamo-nos a testar, apenas, se fatores relacionados ao ambiente socioeconômico de cada município são determinantes na obtençấo dos índices de eficiência. Para isso, os índices foram controlados por variáveis socioeconômicas, usando o modelo Tobit.

A hipótese central deste trabalho é que os índices de eficiência da Polícia Militar, no combate à criminalidade nos municípios mineiros, são bastante heterogêneos, e municípios maiores apresentam menores índices de eficiência, enquanto municípios menores são mais eficientes no combate à criminalidade.

Pode-se argumentar como justificativa à hipótese levantada que, embora a Polícia Militar seja caracterizada por uma estrutura hierárquica, disciplinada e rígida, com centralização quase total de suas decisóes, sua articulação operacional prevê a interação com a comunidade local. Assim, é de se esperar que, em municípios com menor população, a presença e a interação da polícia com a comunidade seja maior do que em municípios com população elevada. ${ }^{2}$

Como será destacado posteriormente, novas formas de arranjos institucionais têm emergido, direcionan- do a ação estratégica da polícia para as especificidades de cada localidade, o que levaria à existência de unidades mais eficientes do que outras no combate à criminalidade. A identificação dessas unidades seria importante, uma vez que estudos mais detalhados poderiam relevar características intrínsecas ao combate à criminalidade nesses municípios, que não são captados por um olhar mais geral do sistema, e que poderiam ser empregados em outros municípios menos eficientes.

Trabalhos relacionados à questão da eficiência no combate ao crime ainda são raros na literatura sobre criminalidade. Carrington et al. (1997) foram um dos primeiros a calcular índices de eficiência técnica no combate à criminalidade da polícia, para o estado australiano de New South Wales, e a investigar a possibilidade de variáveis socioeconômicas influenciarem esses escores. Sun (2002) realizou um estudo semelhante para distritos policiais na cidade de Taipei, em Taiwan, e também verificou se fatores sociais, como, por exemplo, população entre 15-29 anos e tamanho dos distritos, exerceriam influência sobre os índices de eficiência calculados. No Brasil, Mello et al. (2005) calcularam índices de eficiência no combate à criminalidade no Estado do Rio de Ja- 
neiro; no entanto, eles não avançaram na investigação dos possíveis determinantes daqueles índices.

A principal contribuição deste trabalho consiste em lançar luz sobre a questão da eficiência no combate ao crime no Estado de Minas Gerais (Estado que possui elevados índices de crimes violentos) ${ }^{3}$ e direcionar o enfoque da segurança pública para o aumento da eficiência no combate à criminalidade, por meio da construção de um índice de eficiência técnica, que permitisse a comparação entre as diversas unidades da Polícia Militar mineira. A obtenção de resultados positivos, neste sentido, poderia embasar estudos futuros, com o intuito de determinar os coeficientes de eficiência e a proposição de políticas públicas de segurança, com base na identificação e análise individual dos benchmarks, isto é, das unidades de referência dentro do Estado de Minas Gerais.

A segunda seção apresenta uma pequena discussão a respeito da criminalidade e da segurança pública. Os procedimentos e as técnicas utilizadas, bem como a discussão dos resultados, são apresentados nas seçôes três e quatro, respectivamente, e, por fim, na quinta e última seção, são feitas as consideraçōes finais.

\section{O debate sobre criminalidade e segurança pública}

Como discutido anteriormente, muito se tem debatido a respeito de políticas de segurança pública que visem a reduzir a criminalidade a níveis razoáveis. Entretanto, dadas a complexidade do problema e a heterogeneidade dos eventos e dos fenômenos que estão sob o conceito de violência, ocorre grande dificuldade na formulação de políticas públicas, visto que são de ordem cognitiva, pois significam identificar fatores de risco distintos a cada situação.

Desta forma, as propostas para solucionar o problema variam tanto quanto sua definição. De um lado, existe a linha de pensamento da Deterence Theory em criminalidade, ou seja, a teoria da dissuasão que credita às organizaçôes do sistema de justiça criminal a maior parcela no controle da criminalidade: se tivermos uma polícia preparada e eficiente, uma legislação adequada e um complexo penitenciário com vagas suficientes para receber os delinquentes, provavelmente as taxas de criminalidade cairiam.

As evidências empíricas, porém, não têm demonstrado nenhuma relação, de forma conclusiva, entre a ação policial e as taxas de criminalidade; além disso, esse quadro parece ser uma uto-
3 Segundo Waiselfisz (2010),

Minas Gerais, que, com sua taxa de 7,7 homicídios em 100 mil habitantes em 1997, ocupava a $25^{\text {a }}$ posição nacional, em 2007, dez anos depois, com taxa de 20,8 homicídios em 100 mil habitantes, subiu para a $19^{a}$ posiçáo. Esses dados evidenciam o crescimento das taxas de crimes violentos no Estado de Minas Gerais. 
pia para o caso brasileiro. A questão que emerge é a de que a dissuasão se relaciona não ao grau de repressão, mas a um incremento nos custos do crime, além de um incentivo benéfico para o não rime (Beato F., 1999).

De outro lado, existe a linha de pensamento que relaciona as taxas de criminalidade diretamente a fatores sociais e à Lei dos Incentivos, ou seja, o nível de escolaridade, o aperfeiçoamento profissional dos indivíduos, as interaçôes familiares, entre muitos outros, seriam fatores determinantes das taxas de criminalidade (Glaeser et al., 1996; Becker, 1968; Merton, 1959 apud Peixoto et al., 2004).

De forma alternativa, Wilson e Kelling (1982) apontam a desordem física e social de uma comunidade como fatores-chave na determinação das taxas de crimes violentos. Esses autores demonstram sua ideia por meio do problema das "janelas quebradas", ou seja, quando um prédio está com algumas janelas quebradas e ninguém as conserta, as pessoas que as quebraram assumem que ninguém cuida do prédio e acabam, por fim, quebrando mais janelas. No limite, a desordem se instala, e as pessoas passam a cometer crimes mais graves. Em outras palavras, quando a desordem social se instala, a probabilidade de um criminoso ser preso é reduzida. Este, sabendo disso, passará a atuar mais nesses locais, já que tem consciência de que será mais difícil ser denunciado.

No Brasil, recentemente, uma discussão tem aflorado a respeito da integração, ou melhor, a falta de integração funcional das diversas organizaçóes do sistema de justiça criminal. A crítica que emerge é que as polícias, na ponta inicial do sistema, operam de forma desarticulada, acarretando a redundância das atividades das organizaçóes policiais, dualidade de seus comandos, dispersáo de recursos e estratégias de controle interno e externo (Paixão, 1993, apud Beato F., 1999).

De concreto, verifica-se que, em consonância com a maior preocupação dos municípios com a questão da segurança pública, estão surgindo outros tipos de arranjo institucional. Um desses novos arranjos é a aproximação do cidadão com as instituiçôes policiais, por meio de mecanismos como o policiamento comunitário (Ribeiro et al., 2004). Desta forma, tal arranjo permite que o policiamento seja distinto em lugares diferentes, uma vez que as prioridades da polícia e as estratégias de policiamento devem ser ajustadas às necessidades e expectativas dos cidadáos de cada comunidade, que tem o direito náo apenas de 
ser consultada, mas também de participar das decisóes sobre as ações a ser implementadas pela polícia.

Ademais, após um longo período de diversos planos e políticas, o governo federal publicou, em 2003, o novo "Plano Nacional de Segurança Pública”, que inclui uma miríade de açóes a ser realizadas pelos Estados, com o objetivo de tornar as Polícias Civil e Militar e o sistema prisional mais racionais e eficientes no que se refere à efetivação da repressão e dissuasão do criminoso, além de propor medidas de cunho preventivo.

Beato F. (1999) destaca duas políticas que têm funcionado em outros países. Segundo estudo realizado pela Rand Corporation, as políticas de combate à oferta de entorpecentes provocaram declínio no número de pessoas que utilizavam cocaína, sobretudo usuários leves; isso porque o controle da oferta de drogas pela sua repressão tem efeitos principalmente sobre o preço da cocaína. A novidade desse estudo está em não tratar o problema das drogas sob o ângulo exclusivo do tráfico, mas da eficácia dos resultados em combinar programas distintos de controle de drogas tanto na oferta como no consumo.
A Holanda ilustra outra implementação bem-sucedida na área de política de segurança, utilizando abordagens não convencionais para o crime e incluindo uma reformulação de natureza conceitual (Djik, 1997 apud Beato F., 1999). A ideia principal é não reformar indivíduos, o que é extremamente difícil, mas dificultar as condiçóes de ocorrência de crimes, pela manipulação de fatores como disponibilidade de alvos, incremento da vigilância e motivação dos agressores (Felson, 1994 apud Beato F., 1999).

A realidade, entretanto, é que, em Minas Gerais, a última década foi marcada por um crescimento expressivo das taxas de crimes violentos, passando de 187,23 crimes violentos por grupo de 100 mil habitantes em 1997 para 527,66 crimes violentos por 100 habitantes em 2005, ou seja, aumento de 181\% em apenas oito anos. Entretanto, destaca-se que apenas a taxa de crimes violentos contra o patrimônio cresceu aproximadamente $250 \%$ no mesmo período (Scalco, 2007). Isso acarretou mudança do padrão das ocorrências registradas no Estado, verificando-se atualmente maior incidência dos crimes 
violentos contra o patrimônio nas cidades com população superior a $100 \mathrm{mil}$ habitantes (Ribeiro et al., 2004).

Além disso, verifica-se que a distribuição espacial das taxas de criminalidade tem efeito de difusão espacial, ou seja, municípios com altas taxas de criminalidade em média são circundados por municípios vizinhos também com alta taxa de criminalidade, e municípios com baixas taxas de criminalidade são circundados por vivinhos com baixas taxas (Pueeh, 2004; Almeida; Haddad, 2005; Scalco, 2007).

Diante desse contexto e da dificuldade imposta à formulação de políticas eficazes de segurança pública, convém destacar uma observação feita por Beato Filho (1998) de que o combate ao crime por parte das organizaçóes policiais pode perfeitamente prescindir de um diagnóstico de suas "causas" para orientar-se na delimitação de estratégias de combate ao crime. A identificação de padrôes, a distribuição e a evolução das taxas de criminalidade podem ser, entre outros fatores, suficientes para a delimitação de políticas públicas de segurança pública.

É exatamente neste ponto que o trabalho se apoia. Além da identificação de padrôes, distribuição e evolução das taxas de criminalidade, acredita-se que problemas relacionados à eficiência técnica das unidades policiais são fatores determinantes das disparidades observadas entre os municípios mineiros.

\section{3_ Métodos e procedimentos utilizados}

Para calcular o índice de eficiência técnica da Polícia Militar de Minas Gerais no combate à criminalidade, foi utilizada a técnica de Data Envelopment Analysis (DEA). Essa técnica fornece um meio de comparação entre os municípios, visto que permite ordená-los segundo um índice de eficiência multidimensional. Os índices calculados são medidas relativas, determinadas pela comparação do alcance de determinado objetivo, dado um conjunto de "insumos", com o objetivo "ideal", com os mesmos níveis de insumos.

$\mathrm{O}$ que se tem em mente é que o cálculo de uma medida comparável de eficiência entre as unidades da Polícia Militar se torna uma tarefa complexa no momento em que esse índice pode refletir características intrínsecas a cada unidade que não estâo relacionadas à ação realizada pela polícia. A priori, comparar a Polícia Militar da capital mineira, Belo Horizonte, com população superior a dois milhóes de habitantes, com o mu- 
nicípio de Ubaí, por exemplo, localizado na Região Norte do Estado e com população aproximada de 10 mil habitantes, seria uma atitude equivocada se não fossem considerados tais problemas. ${ }^{4}$

Neste sentido, é necessário testar se as diferenças encontradas nos índices calculados são reflexos de características realmente relacionadas à eficiência técnica das diversas unidades da Polícia Militar ou se são influenciados pelo ambiente socioeconômico encontrado em cada município.

\section{1_ Obtenção das medidas de eficiência: "Data Envelopment Analysis" (DEA)}

As medidas de eficiência podem ser facilmente obtidas de problemas simples que abrangem poucos insumos e produtos. Entretanto, em situaçóes em que várias unidades utilizam múltiplos insumos e produzem vários produtos, o cálculo da eficiência relativa de cada unidade tornase mais complexo. Para solucionar essas dificuldades, podem-se obter, com base em uma amostra de dados, fronteiras eficientes, que servirão como referencial para as comparaçôes entre as unidades (Gomes et al., 2004).

As fronteiras podem ser estimadas por diferentes métodos - paramétricos ou não paramétricos. As fronteiras estocásticas são abordagens paramétricas, sendo estimadas por métodos economé- tricos, enquanto a análise envoltória de dados (DEA) é uma abordagem não paramétrica, que envolve programação matemática em sua estimação. Nesse sentido, Charnes et al. (1978) propuseram o primeiro modelo que ficou conhecido como Data Envelopment Analysis (DEA).

A ideia central da Análise Envoltória de Dados é encontrar a melhor DMU (ou unidade de tomada de decisão) virtual para cada DMU real. Se a DMU virtual, que pode ser obtida por meio de uma combinação convexa de outras DMUs reais, conseguir produzir maiores quantidades de produtos, utilizando-se da mesma ou de menor quantidade de insumos, então a DMU real será ineficiente. As unidades eficientes que, quando combinadas, fornecem a DMU virtual para a unidade ineficiente são conhecidas como pares ou benchmarks daquela DMU.
4 É de se pensar que a polícia da capital mineira enfrenta uma "realidade" completamente diferente da pequena cidade mineira de Ubaí e que essas diferenças teriam impacto significativo no resultado da eficiência técnica calculada para cada um desses municípios. Seria precipitado, porém, tomar tal justificava como único argumento para a não utilização de um índice de eficiência que os compare, já que fatores como a disponibilidade e/ou alocação de recursos, estratégias de atuação ou o envolvimento da polícia com a sociedade local também são os que determinam a eficácia de qualquer política de combate à criminalidade. 
5 Existem também os modelos mistos (mixed models) que nâo são descritos aqui. Para mais detalhes, podese consultar Charnes et al. (1994), Cooper et al. (2000) e Lins; Meza, (2000).

6 Nos modelos com orientação ao insumo, a medida de eficiência $(\theta)$ é menor ou igual à unidade, indicando a máxima redução na utilização dos insumos, mantendo-se fixas as quantidades dos produtos. De maneira análoga, em um modelo com orientação ao produto, a medida de eficiência $(\varphi)$ é maior ou igual à unidade, indicando a máxima expansão da produção, mantendo-se fixas as quantidades dos insumos.
$\mathrm{Na}$ literatura, as duas principais formas de calcular a eficiência técnica das DMUs são os modelos orientados a insumos e os modelos orientados a produto. ${ }^{5}$ Aqueles buscam identificar a ineficiência técnica das DMUs mediante redução proporcional na utilização dos insumos, ao passo que esses buscam obter medidas de eficiência com o aumento proporcional na produção, mantendo-se as quantidades de insumos fixas. ${ }^{6}$

Nos modelos com orientação produto, a medida de eficiência, $\varphi$, assume um valor unitário apenas quando a DMU é eficiente, indicando que, nesse caso, não há possibilidade de expansão dos produtos, mantendo-se fixas as quantidades de insumos. O problema com orientação produto, pressupondose retornos constantes à escala, pode ser escrito da seguinte forma:

\section{$\operatorname{MAX}_{\varphi, \lambda} \varphi$,}

sujeito a:

$$
\begin{aligned}
& -\varphi y_{i}+Y \lambda \geq 0, \\
& x_{i}-X \lambda \geq 0, \\
& \lambda \geq 0 .
\end{aligned}
$$

em que $1 \leq \varphi<\infty$ e $\varphi-1$ é o aumento proporcional nos produtos que poderiam ser obtidos pela i-ésima
DMU, mantendo-se constante a utilização de insumos. A medida de eficiência técnica seria dada por $1 / \varphi$, que varia de zero a um.

O modelo de retornos constantes à escala pode ser reformulado com o objetivo de possibilitar retornos variáveis às DMUs analisadas. Essa proposta foi inicialmente feita por Banker et al. (1984), cujo modelo ficou conhecido como BCC, por causa das iniciais do nome dos autores. A ideia é introduzir uma restrição de convexidade ao modelo CCR (retornos constantes) apresentado em (1). O modelo BCC, que pressupóe retornos variáveis à escala, pode ser representado pela seguinte notação algébrica:

$M A X_{\varphi, \lambda} \varphi$

sujeito a:

$$
\begin{aligned}
& -\varphi y_{i}+Y \lambda \geq 0, \\
& x_{i}-X \lambda \geq 0, \\
& N_{1} \lambda=1 \\
& \lambda \geq 0 .
\end{aligned}
$$

em que N1 é um vetor (n x 1) de algarismos unitários $(1, \ldots, 1)$. Essa abordagem forma uma superfície convexa de planos em interseçấo, que envolve os dados de forma mais compacta do que a superfície formada pelo modelo com retornos constantes. 


\section{2_ Teste dos determinantes do índice de eficiência}

Para testar a hipótese de que fatores socioeconômicos exerceriam influência na determinação dos escores calculados, foi utilizado o modelo econométrico de análise censurada Tobit. Análises que utilizam da técnica DEA e Regressão Tobit são frequentes em artigos acadêmicos. Alguns exemplos são os trabalhos de Kirjavainen e Loikkanen (1998), que avaliaram a eficiência de 291 escolas secundárias da Finlândia; de Shao e Lin (2002), que investigaram os efeitos da Tecnologia de Informação (TI) na eficiência de 370 empresas; de Sun (2002), que realizou um trabalho semelhante a esse para a cidade de Taipei, em Taiwan; e o trabalho de Marinho (2003), que avaliou a eficiência dos serviços ambulatoriais e hospitalares nos municípios do Estado do Rio de Janeiro.

O modelo Tobit, de acordo com Greene (2008), é utilizado nos casos em que a variável dependente está limitada entre faixas de valores ou concentrada em pontos iguais a um valor-limite. Nesses casos, ocorre o que se chama na literatura econométrica de "amostra censurada”. A aplicação do modelo Tobit busca contornar o problema da censura valendo-se de técnicas estatísticas que possibilitem fazer inferências para toda a população sem perda de qualidade, como ocorria no caso de variáveis truncadas.

As variáveis explicativas do modelo Tobit, segundo Greene (2008), podem ser definidas pelo vetor $X=\left(x_{1}, x_{2}, \ldots, x_{p}\right)$, e a variável de resposta, definida por $\mathrm{Y}^{*}$, representando o Y observado por meio dos escores de eficiência da abordagem DEA. O modelo econométrico pode ser formulado da seguinte maneira: $y_{i}^{*}=X_{I} \beta+u_{i}$, em que se observa efetivamente a variável y, sendo u o erro aleatório da equação. O valor da censura é denotado por $y_{i}^{c}$, com o subscrito i indicando a censura em cada observação; o que se observa na amostra é:

$\left\{\begin{array}{l}y_{i}=y_{i}^{*} \text { se } y_{i}^{*}<y_{i}^{c} \\ y_{i}=y_{i}^{c} \text { se } y_{i}^{*} \geq y_{i}^{c}\end{array}\right.$

De acordo com Ferreira e Braga (2007), a estimação dos parâmetros $\beta$ 's pelo método de MQO (mínimos quadrados ordinário) resultaria em parâmetros viesados, uma vez que a censura introduz correlação entre o termo do erro da regressão e as covariadas (X's). Dessa forma, a esperança do erro deve ser calculada como uma esperança condicional, representada por:

$E\left[u_{i} \mid u_{i}<y_{i}^{c}-X_{I} \beta\right]$. 
Uma alternativa para a obtenção de estimadores consistentes seria a de calcular os parâmetros $\beta$ 's do modelo de regressão por meio do método de máxima verossimilhança. Esse método permite isolar e descrever a contribuição das variáveis censuradas, bem como das variáveis não censuradas na formação $\mathrm{da}$ função de verossimilhança.

Vale observar que os índices de eficiência estão localizados entre zero e um; portanto, tomou-se o inverso deles, que passaram, então, a estar situados entre um e infinito, sendo censurados à esquerda em um.

\section{3_Dados e procedimentos utilizados}

Os dados sobre criminalidade foram obtidos na Fundação João Pinheiro, cuja base foi elaborada valendo-se dos dados primários da Polícia Militar de Minas Gerais, para o ano de 2005. As variáveis utilizadas no cálculo das taxas de criminalidade foram agrupadas em dois grupos distintos, como frequentemente feito em pesquisas da área: o primeiro grupo corresponde aos crimes violentos contra a pessoa (CVPE), composto das ocorrências de homicídio consumado, homicídio tentado e estupro; o segundo corresponde aos crimes violentos contra o patrimônio (CVPA), composto das ocorrências de roubo e roubo à mão armada.
Em vários estudos sobre criminalidade, a principal razão para diferenciar crimes contra a pessoa e contra o patrimônio está em verificar se as pessoas que praticam esses crimes têm motivaçáo diferenciada em relação à sua prática. De maneira geral, os crimes violentos contra pessoas estão, sobretudo, relacionados com motivação náo necessariamente ligada a fatores puramente econômicos, mas sim relacionados à desagregação familiar e à influência do grupo. Os crimes contra o patrimônio, por sua vez, estão mais relacionados a fatores de natureza econômica.

Para superar problemas associados à utilização de tais variáveis em pesquisa criminológica, como, por exemplo, a comparação da ocorrência de eventos entre diferentes populaçóes ou na mesma população em momentos diferenciados no tempo, o número de ocorrências foi ponderado pela população e agrupado em grupos de 100 mil habitantes (taxa bruta). Contudo, ainda assim, persistem problemas relacionados à alta instabilidade gerada por localidades de tamanhos diferentes.

Desta forma, foram estimadas as taxas de crimes violentos contra a pessoa $\left(\mathrm{CVPE}^{*}\right)$ e crimes violentos contra o patrimônio $\left(\mathrm{CVPA}^{*}\right)$, utilizando-se um método estatístico bayesiano proposto 
por Marshall (1991). A ideia central desse método é usar o risco contido nos dados das outras áreas para estimar o risco de certa área específica, diminuindo de maneira considerável o efeito das flutuaçóes aleatórias não associadas ao risco. ${ }^{7}$

O fundamental é corrigir as taxas usuais (taxas brutas) para obter uma nova taxa que seja estimada com menos variabilidade quando a população é pequena. Num município qualquer, a estimativa $\theta$ da taxa corrigida é calculada como uma média ponderada da taxa bruta $(t)$ e uma taxa média $(m)$ de todo o Estado:

$\theta_{i}=c_{i} t_{i}+\left(1-c_{i}\right) m$

em que $c_{i}=\frac{s^{2}-m / \bar{n}}{s^{2}-m / \bar{n}+m / n_{i}}, m$ é a

taxa média do Estado, $\bar{n}$ é a população

média, $n_{i}$ é a população observada no município $i, s^{2}=\sum_{i} \frac{n_{i}\left(t_{i}-m\right)^{2}}{n}, n$ é a população total do Estado, e t é a taxa bruta observada no município $i$. O valor de $c_{i}$ varia de município para município num intervalo dado $[0,1]$ e é o peso associado à taxa bruta em comparação com o peso associado à taxa média. Quando $c_{i}$ for um valor próximo de 1 , o valor de $\theta$ será próximo do valor da taxa bruta $t_{i}$. Quando $c_{i}$ for próximo de 0 , o valor $\theta$ será aproximadamente igual a $m$.

Em síntese, a taxa bayesiana empírica global é uma média ponderada entre a taxa bruta da localidade e a taxa global. Se a localidade apresentar população considerável, sua taxa apresentará pequena variabilidade e ela permanecerá praticamente inalterada. Se, por outro lado, a localidade apresentar população pequena, a estimativa da taxa bruta terá grande variância e pouco peso será atribuído a essa taxa instável, tornando a taxa bayesiana mais próxima do valor esperado de uma área escolhida ao acaso naquela regiáo.

Para o cálculo dos índices de eficiência técnica, isto é, as DMUs, foram empregados dados de 851 municípios, em razão da falta de informações referentes aos municípios de Capim Branco e São Sebastião do Anta. A utilização de municípios como unidade tomadora de decisôes deve-se, basicamente, a duas razões: primeiro, os dados em nível de município são a máxima desagregação disponível, e, segundo, com o surgimento de outros arranjos institucionais, como o policiamento comunitário, por exemplo, tem-se maior interação da polícia com a sociedade. Isso permite definir estratégias apropriadas para cada localidade,
O método proposto por Marshall (1991) é bastante simples de ser implementado e possui a vantagem de fácil integração a ambientes de geoprocessamento, estando disponível em diversos deles, como TerraVIew, GeoDa, entre outros. 
8 É importante destacar que esses náo seriam os únicos insumos utilizados no sistema de segurança pública. Ter-seia um conjunto mais amplo de insumos, como veículos, sistemas de monitoramento eletrônico, armamentos, recursos financeiros, etc.; entretanto, tais variáveis não foram disponibilizadas para a realização deste trabalho.

9 Para o cálculo dos índices de eficiência, foi utilizado o software DEAP, versão 2.1. mesmo sendo a Polícia Militar uma instituição rígida e hierárquica quanto à tomada de decisóes.

As seguintes variáveis foram utilizadas no modelo de Análise Envoltória de Dados para calcular os índices de eficiência:

Insumo (input)

$x_{1}$ - número de policiais militares por grupo de 1.000 habitantes; $^{8}$

Produto (output)

$y_{1}$ - número de prisóes registradas de crime violento contra a pessoa por policial;

$y_{2}$ - número de prisóes registradas de crime violento contra o patrimônio por policial;

$y_{3}$ - inverso da taxa de crimes violentos contra a pessoa $\left(\mathrm{CVPE}^{*}\right)$; e

$y_{4}$ - inverso da taxa de crimes violentos contra o patrimônio $\left(\mathrm{CVPA}^{*}\right)$.

Os índices foram calculados pressupondo retornos variáveis à escala e utilizando a orientação produto. ${ }^{9}$

A primeira pressuposição foi feita para incorporar as propriedades relacionadas ao tamanho dos municípios, sendo esperado que a contribuição marginal de mais um policial, por exemplo, seja maior em municípios pequenos do que a contribuição marginal gerada em mu- nicípios grandes. A utilização do modelo de retornos variáveis incorpora questôes relacionadas ao ganho de escala.

O emprego do modelo de orientação produto deve-se ao fato de que o número de policiais existentes em cada município é relativamente fixo. A construção de residências fixas e restriçooes legais, por exemplo, dificulta a realocação desses policiais entre os municípios. Além disso, a análise com orientação produto permite identificar a produção potencial das DMUs; assim, o índice de eficiência seria uma referência para identificar fatores que poderiam elevar "produção" de outras unidades tidas como ineficientes.

Outro ponto que merece ser destacado diz respeito a uma deficiência do modelo calculado, pois um dos pressupostos do modelo DEA é que as DMUs sejam homogêneas, característica não observada entre os municípios analisados. Para contornar tal problema, todas as variáveis utilizadas no modelo são do tipo intensivas (variáveis que são divididas por algum indicador de intensidade, como população, por exemplo), amenizando o problema de heterogeneidade entre as DMUs.

$\mathrm{Na}$ análise dos fatores que poderiam ter impacto sobre índice de eficiência calculado, trabalhos como os 
de Becker (1968), Cohen; Felson (1979), Wilson; Kelling (1982), Glaeser et al. (1996), Beato F. (1998), Araújo; Fajnzylber (2000), Messner; Anselin (2004), Pueeh (2004), Mello et al. (2005) e Levitt; Dubner (2005) foram utilizados para identificar um conjunto de variáveis que são normalmente usadas para representar o ambiente socioeconômico das localidades e que teriam impacto significativo na determinação das taxas de criminalidade.

Neste sentido, dentro de um conjunto de 19 variáveis identificadas, puderam-se utilizar conjuntamente nove delas. ${ }^{10}$ Além disso, foram empregadas variáveis dummies para identificar três grupos de municípios, de acordo com o seu tamanho. As variáveis utilizadas para representar o ambiente socioeconômico dos municípios foram coletadas no Atlas de Desenvolvimento Humano e dizem respeito à $^{11}$ : densidade demográfica (DD); percentual da população urbana (PU); Índice de Gini (IG); taxa bruta de frequência à escola (TBFE); percentual de mulheres chefes de família (PMCF); renda per capita (RP); percentual de domicílios sem banheiro e água encanada (PDSBA); percentual de adolescentes fora da escola em 1991 (PAFE); percentual de crianças fora da escola em 1991 (PCFE); municípios com população inferior a 10.000 habitantes (D1); e mu- nicípios com população entre $10.001 \mathrm{e}$ 50.000 habitantes (D2).

A hipótese subjacente a esse teste é a de que os escores de eficiência são determinados por características socioeconômicas de cada município. Caso a hipótese não seja rejeitada, por exemplo, seriam esperados escores de eficiência maiores em municípios com fatores socioeconômicos mais favoráveis do que municípios com fatores socioeconômicos mais degradados. Caso contrário, restaria testar a hipótese de que os escores de eficiência seriam determinados por fatores relacionados à função de policiamento ostensivo, que, se confirmada, validaria a utilização de índice como instrumento de auxílio no combate à criminalidade. ${ }^{12}$

\section{Algumas variáveis foram excluídas do modelo por serem altamente correlacionadas, e outras variáveis não foram utilizadas por causa da indisponibilidade de dados. \\ 11 As estatísticas descritivas das variáveis utilizadas na estimação do modelo Tobit encontram-se na Tabela A, em anexo.}

\author{
12 A indisponibilidade de \\ informaçôes, principalmente \\ relacionadas à função de \\ policiamento, como o \\ número de viaturas, gastos \\ com combustíveis, sistemas \\ de monitoração eletrônica, \\ entre outros, impossibilitou \\ testar se fatores como a \\ disponibilidade e/ou alocação \\ de insumos utilizados nessa \\ função seriam determinantes \\ para os valores dos índices \\ obtidos. Por isso, os testes de \\ hipóteses limitaram-se apenas \\ às variáveis socioeconômicas.
}




\section{4_Resultados e discussão}

\section{1_Análise da eficiência}

Os índices de eficiência técnica da Polícia Militar, no combate à criminalidade, para os municípios mineiros são apresentados na Figura 1. Esse mostra que os municípios mineiros estão distribuídos assimetricamente à direita, em torno da média $(0,49)$, e existe uma concentração de municípios (636) entre as faixas de eficiência de 0,3 a 0,6. Do total de municípios, apenas 14 se mostraram 100\% eficientes (apresentaram índice de eficiência igual a 1) e 35 apresentaram índice de eficiência calculado superior a 0,9 .
A Tabela 1 mostra a distribuição dos municípios mineiros em quatro estratos, de acordo com os índices de eficiência. Os municípios foram divididos com eficiência entre 0 e 0,25 (baixa eficiência); 0,25 e 0,50 (eficiência regular); 0,50 e 0,75 (eficiência média); e 0,75 e 1,00 (alta eficiência). Para cada estrato, são apresentados os valores médio, máximo e mínimo de cada variável utilizada, bem como o tamanho da população e o número de observaçóes em cada grupo.

De modo geral, verifica-se que a distribuição dos municípios entre os quatro estratos ocorre de acordo com a lógica do modelo de eficiência, ou seja, em

\section{Figura 1 _ Histograma da distribuição dos índices de eficiência}

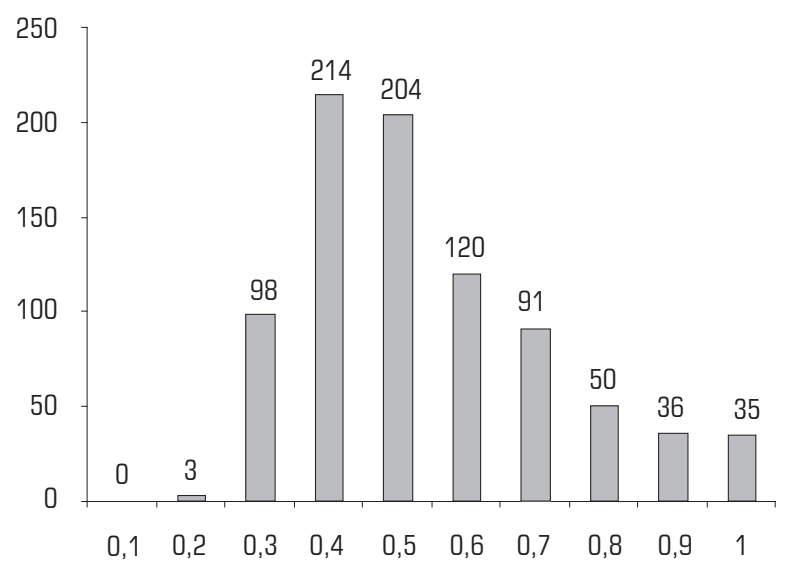

Fonte: Elaboração própria. 
Tabela 1_Resultados da Análise Envoltória de Dados - DEA

\begin{tabular}{|c|c|c|c|c|c|c|}
\hline \multirow[t]{2}{*}{ Variáveis } & & \multicolumn{4}{|c|}{ Estratos de Eficiência } & \multirow[t]{2}{*}{ Média Geral } \\
\hline & & $0-0,25$ & $0,25-0,50$ & $0,50-0,75$ & $0,75-1,00$ & \\
\hline \multirow[t]{3}{*}{ POLICIAL / 1.000 hab. } & Média & 1,51 & 1,17 & 0,82 & 0,61 & 1,03 \\
\hline & Máximo & 4,57 & 8,52 & 4,01 & 2,72 & \\
\hline & Mínimo & 0,49 & 0,18 & 0,11 & 0,09 & \\
\hline \multirow[t]{3}{*}{ CVPE* } & Média & 93,01 & 60,94 & 43,48 & 33,21 & 54,60 \\
\hline & Máximo & 130,70 & 166,40 & 117,30 & 81,40 & \\
\hline & Mínimo & 66,40 & 33,00 & 23,10 & 16,80 & \\
\hline \multirow[t]{3}{*}{$\mathrm{CVPA}^{*}$} & Média & 123,85 & 66,06 & 47,23 & 44,08 & 61,23 \\
\hline & Máximo & 998,50 & 1079,70 & 319,90 & 254,50 & \\
\hline & Mínimo & 17,00 & 5,40 & 4,10 & 2,20 & \\
\hline \multirow[t]{3}{*}{ Prisóes / Policial CVPE } & Média & 1,28 & 2,43 & 4,72 & 6,75 & 3,49 \\
\hline & Máximo & 4,00 & 10,50 & 16,00 & 25,71 & \\
\hline & Mínimo & 0,00 & 0,00 & 0,00 & 0,00 & \\
\hline \multirow[t]{3}{*}{ Prisóes / Policial CVPA } & Média & 0,44 & 0,73 & 1,42 & 1,87 & 1,03 \\
\hline & Máximo & 1,36 & 3,47 & 6,00 & 11,00 & \\
\hline & Mínimo & 0,00 & 0,00 & 0,00 & 0,00 & \\
\hline \multirow[t]{3}{*}{ População } & Média & 74.451 & 19.174 & 18.040 & 17.022 & 21.350 \\
\hline & Máximo & 2.274 .790 & 546.733 & 187.866 & 137.824 & \\
\hline & Mínimo & 1.766 & 887 & 1.372 & 3.316 & \\
\hline Observaçóes & & 42 & 477 & 240 & 92 & 851 \\
\hline
\end{tabular}

Fonte: Elaboraçấo própria.

média, os municípios mais eficientes foram os que apresentaram menor número de policiais por grupo de 1.000 habitantes, além de menores taxas de CVPE* e CVPA*, e maior taxa de prisóes por policial, para crimes violentos tanto contra a pessoa quanto contra o patrimônio. Além disso, nota-se que, em média, são os menores municípios aqueles que possuem os maiores escores de eficiência.

Para verificar, com maior rigor se há diferenças nas medidas de eficiência entre os municípios maiores e menores, também foram utilizados os testes não paramétricos U de Mann-Whitney e o teste W de Wilcoxon. 
Tabela 2_Testes de igualdade de fronteira de Mann-Whitney e Wilcoxon

\begin{tabular}{l|c|c|c}
\multirow{2}{*}{ Grupos } & \multicolumn{2}{|c}{ Medida de pura eficiência técnica } & $\begin{array}{c}\text { \% de municípios com } \\
\text { máxima eficiência }\end{array}$ \\
\cline { 2 - 4 } & Média & Coeficiente de variação (\%) & 4,13 \\
\hline 2 & 0,8032 & 11,30 & 5,30 \\
\hline
\end{tabular}

U de Mann-Whitney: 18.849,5

W de Wilcoxon: 148.135,5

Significância: 0,000

\begin{tabular}{l|c|c|c}
\hline 1 & 0,9902 & 1,16 & 19,69 \\
\hline 3 & 0,8466 & 12,46 & 3,33 \\
\hline
\end{tabular}

U de Mann-Whitney: 1.024,0

W de Wilcoxon: 2.854,0

Significância: 0,000

\begin{tabular}{l|c|c|c}
\hline 2 & 0,9907 & 1,37 & 18,37 \\
\hline 3 & 0,8844 & 12,33 & 8,33 \\
\hline
\end{tabular}

U de Mann-Whitney: 2.050,5

W de Wilcoxon: 3.880,5

Significância: 0,000

Fonte: Elaboraçăo própria.

O teste de Mann-Whitney objetiva verificar se duas amostras independentes pertencem à mesma população, sendo aplicado quando se pode supor um grau razoável de independência das operaçôes das DMUs, tomadas individualmente. Quando a medida de eficiência for relativa e obtida por comparação, deve-se recorrer ao teste de Wilcoxon W, recomendado para amostras dependentes.
Seguindo o método empregado por Marinho e Façanha (2000), os municípios foram separados em três grupos, dependendo do tamanho da população, ${ }^{13}$ e os testes foram aplicados considerando-se dois grupos separadamente, ou seja, grupos 1 e 2; grupos 1 e 3; e grupos 2 e 3 .

Os resultados da Tabela 2 indicam que, em todos os pares de grupos comparados, tanto o teste de Mann-Whitney 
quanto o teste de Wilcoxon rejeitaram a hipótese nula da igualdade entre as fronteiras. Nesse sentido, a relação entre nível de eficiência e tamanho do município não é rejeitada pelos testes.

Esses resultados confirmam a hipótese levantada na seção inicial do trabalho. Além da heterogeneidade dos municípios, verificada pela distribuição entre os estratos identificados, o problema, no que concerne à eficiência ou à ineficiência, está diretamente relacionado ao tamanho dos municípios.

Entretanto, é importante salientar que existem municípios em meio a esses estratos que são casos especiais e são importantes, portanto, para a avaliação deste estudo. Os municípios de Poços de Caldas e Varginha, por exemplo, são os únicos entre os 23 com população superior a 100.000 habitantes, no Estado mineiro, localizados no estrato de alta eficiência $(0,75-1,00)$.

Poços de Caldas possui taxas de CVPE* $^{*}$ e CVPA* iguais a 26,90 e 154,80, respectivamente, e elas são $64 \%$ e $62 \%$, respectivamente, inferiores à taxa média dos municípios desse porte. Além disso, o número de policiais por grupo de mil habitantes é 1,78, (25\% inferior à média desses municípios), e suas taxas de prisão por policial, tanto para crimes violentos contra a pessoa quanto contra o patrimônio, são 6,50 e 2,20, respectivamente (esses valores correspondem a taxas de prisão $67 \%$ e $39 \%$, respectivamente, superiores à taxa média desses municípios). Tais variáveis destacam suas características e justificam o fato de serem apenas esses dois municípios, com população superior a 100 mil habitantes, em meio ao estrato de alta eficiência, que demonstram o porquê do alto escore de eficiência calculado $(0,792$ e 0,93, respectivamente).

A análise dos índices de eficiência indicou que, embora a Polícia Militar seja caracterizada por uma estrutura hierárquica, disciplinada e rígida, idealizada pela concepção de seus membros mais graduados, com uma centralização quase total de suas decisões estratégicas, ela apresenta diferenças significativas entre os 851 municípios mineiros analisados. As diferenças observadas nos escores de eficiência da Polícia Militar podem ser reflexos de formas alternativas de gerenciamento e utilização de insumos (policiais), como planejamento estratégico, por exemplo, que cada unidade determina. Entretanto, elas também podem ser resultado de diferentes ambientes sociais enfrentados pela PM. 
14 Para mais detalhes, ver Becker (1968), Cohen; Felson (1979), Wilson; Kelling (1982), Glaeser et al. (1996), Beato F. et al. (1998), Araújo; Fajnzylber (2000), Messner; Anselin (2004), Pueeh (2004), Mello et al. (2005) e Levitt; Dubner (2005).

\section{2_Determinantes dos escores de eficiência técnica}

Como discutido na seção anterior, tornase necessário verificar quais fatores seriam determinantes dos diferentes índices de eficiência da Polícia Militar nos municípios mineiros. Os resultados obtidos pelo DEA, na Tabela 1, forneceram indícios de que municípios maiores possuem índices de eficiência menores e que esse padrão poderia estar relacionado a características intrínsecas a cada município.

Portanto, o próximo passo consistiu em testar a hipótese de que os índices de eficiência técnica da Polícia Militar seriam, ou não, determinados pelo ambiente socioeconômico encontrado em cada município analisado. Para isso, utilizou-se o modelo econométrico de análise censurada Tobit, que permite verificar a capacidade de explicação da variabilidade dos índices calculados, controlando a variável dependente (índice de eficiência) pelas variáveis explicativas determinadas na seção 3.2.

Os resultados da relação entre índices de eficiência e as variáveis explicativas são apresentados na Tabela 3. Uma observação interessante das estimativas está relacionada à significância estatística dos coeficientes e à comparação entre os sinais esperados a priori e os obtidos pela estimativa.
Do ponto de vista estatístico, os coeficientes relacionados às variáveis densidade demográfica (DD), percentual da população urbana (PU), percentual de mulheres chefes de família (PMCF) e percentual de domicílios sem banheiro e água encanada (PDSBA) foram estatisticamente diferentes de zero, ao nível de 5\% de significância, na explicação da variabilidade dos escores de eficiência.

Quanto aos sinais dos coeficientes, de maneira geral, verifica-se que as variáveis significativas no modelo apresentaram sinais como esperados a priori. De acordo com os trabalhos utilizados como referência, ${ }^{14}$ as evidências indicam relação direta entre densidade demográfica (DD), percentual da população urbana (PU) e níveis de criminalidade. Essa relação poderia ser explicada por maiores oportunidades de crime. Neste caso, poder-se-ia afirmar que, quanto maiores a parcela da população urbana (PU) e a densidade demográfica (DD), maior a quantidade de "alvos" potenciais e maiores seriam as oportunidades para ação criminosa, o que acarreta, consequentemente, maior dificuldade no controle da criminalidade. Portanto, afeta negativamente a medida de eficiência técnica da Polícia Militar nesses locais.

O percentual de domicílios sem banheiro e água encanada (PDSBA) foi 
Tabela 3_Fatores associados à eficiência técnica da Polícia Militar no combate à criminalidade, para os municípios de Minas Gerais

\begin{tabular}{|c|c|c|c|c|}
\hline Variáveis & Coeficientes & Erro padrão & Estatística Z & Prob. \\
\hline $\mathrm{C}$ & 1,206931 & 0,962461 & 1,254005 & 0,2098 \\
\hline DD & 0,000322 & 0,000107 & 3,020406 & 0,0025 \\
\hline PU & 0,70336 & 0,213852 & 3,288998 & 0,001 \\
\hline IG & 0,727405 & 0,712319 & 1,021179 & 0,3072 \\
\hline TBFE & 0,172735 & 0,80555 & 0,214432 & 0,8302 \\
\hline PMCF & 0,728879 & 0,331646 & 2,197759 & 0,028 \\
\hline RP & $-0,00083$ & 0,000749 & $-1,10444$ & 0,2694 \\
\hline PDSBA & 11,74514 & 4,736742 & 2,479582 & 0,0132 \\
\hline PAFE & $-0,87587$ & 0,472876 & $-1,85223$ & 0,064 \\
\hline PCFE & 1,171259 & 0,746081 & 1,569882 & 0,1164 \\
\hline D1 & 0,271084 & 0,142271 & 1,905404 & 0,0567 \\
\hline D2 & $-0,08139$ & 0,134545 & $-0,60496$ & 0,5452 \\
\hline Log likelihood & $-1,2224$ & & & \\
\hline$x^{2 ¥}$ & 8,25763 & & & 0,000 \\
\hline $\mathrm{R}^{2}$ & 0,0938 & & & \\
\hline
\end{tabular}

Notas: A variável dependente é o inverso do índice de eficiência (ou seja, $1 / \varphi$ ). DD: Densidade demográfica; PU: Percentual da população urbana; IG: Índice de Gini; TBFE: Taxa bruta de frequência à escola; PMCF: Percentual de mulheres chefes de família; RP: Renda per capita; PDSBA: Percentual de domicílios sem banheiro e água encanada; PAFE: Percentual de adolescentes fora da escola, em 1991; PCFE: Percentual de crianças fora da escola, em 1991; D1 = 1 para populaçáo inferior a 10.000 habitantes e igual nos demais casos. $\mathrm{D} 2=1$ para população entre 10.001 e 50.000 habitantes; $=0$ demais casos $¥$ Teste de restrição $H 0: D D=P U=I G=T B F E=P M C F=R P=P D S B A=P A F E=P C F E=0$. H1: pelo menos um dos coeficientes é diferente de zero.

utilizado como uma proxy para mensuração de residências com baixos índices de saneamento básico (habitaçóes típicas de regióes menos favorecidas economicamente, como favelas, por exemplo), locais onde se concentra grande parte de criminosos e onde é intensa a comercialização de entorpecentes. Novamente, o sinal encontrado é o mesmo esperado $a$ priori, ou seja, quanto maior a quantidade de domicílios desse tipo, maiores a taxas de criminalidade e, consequentemente, maior é a dificuldade no controle de tal problema, afetando negativamente o índice de eficiência.

Outro resultado relevante é o encontrado por meio da variável percentual de mulheres chefe de família (PMCF). 
Essa variável visa a captar, de certa forma, o que alguns autores chamam de “desorganização social”. Segundo Araújo Jr. e Fanjnzylber (2000), a hipótese de que essa variável teria um efeito potencial sobre o crime vem da ideia de que vínculos interpessoais sólidos contribuiriam para gerar barreiras à atividade criminal, na medida em que seriam fontes de controle e proteção contra a violência. Assim, verificou-se um sinal positivo, indicando que municípios com maior percentual de mulheres chefes de família (PMCF) tenderiam a ter índices de eficiência menores.

Por fim, contrários ao esperado a priori, porém, significativos ao nível de $10 \%$ de significância, encontram-se as variáveis percentual de adolescentes fora da escola em 1991 (PAFE) e a variável dummy correspondente a municípios pequenos, com população inferior a 10.000 habitantes (D1). A priori, esperava-se uma relação negativa entre a primeira variável e o índice de eficiência, já que, de acordo com a literatura sobre criminalidade, pessoas com menores condições escolares seriam mais propensas a ingressar em atividades criminosas. Desta forma, representados pelo percentual de adolescentes fora da escola em 1991, hoje, já adultos, estariam diretamente correlacionados com maiores taxas de criminalidade e, consequentemente, com menores índices de eficiência. Da mesma maneira, era esperado que em municípios menores a interação da polícia com a sociedade fosse maior, o que resultaria num ambiente mais propício para o combate à criminalidade e em maiores índices de eficiência.

Feita a descrição individual das variáveis, destaca-se que a principal inferência feita, com base nos resultados, diz respeito à capacidade de essas variáveis explicarem, conjuntamente, a variabilidade dos índices de eficiência técnica calculados. Verifica-se, por meio do coeficiente de determinação da equação ajustada $\left(\mathrm{R}^{2}\right)$, que o modelo utilizado explica apenas, aproximadamente, $10 \%(0,0938)$ da variabilidade dos índices de eficiência. Esses resultados se assemelham àqueles obtidos por Carrington et al. (1997) e Sun (2002), em que os coeficientes obtidos foram 0,066 e 0,061, respectivamente.

Embora seja um resultado contrário ao comumente buscado em métodos estatísticos de regressão, o baixo valor do coeficiente de determinação da equação permite tirar uma importante conclusão dos resultados. Assim como em Carrington et al. (1997) e Sun (2002), podese afirmar que as variáveis utilizadas no 
teste de determinação dos índices de eficiência técnica, ainda que significativas, explicam pouco daquela variável. Em outras palavras, pode-se concluir que o ambiente socioeconômico dos municípios não exerce muita influência sobre os índices de eficiência técnica calculados.

Desta forma, conforme discutido na seção inicial do trabalho, mesmo que não pareça muito adequado comparar, por exemplo, a eficiência técnica das polícias de Belo Horizonte e Ubaí, a análise demonstra que a diferença entre os níveis de eficiência técnica das duas polícias $(0,241$ e 1,00, respectivamente) pode ser reflexo de diferenças existentes na própria eficiência da polícia desses municípios, e não a aspectos socioeconômicos dessas duas cidades.

Portanto, os resultados obtidos sinalizam para o fato de que o estudo desses aspectos, diretamente relacionados à eficiência técnica, pode se tornar fundamental na determinação de políticas públicas de combate à criminalidade, uma vez que fica evidente que existem unidades da Polícia Militar que são mais eficientes do que outras no combate à criminalidade e que tais diferenças não são, a priori, reflexo do ambiente socioeconômico encontrado em cada município.

\section{5_Conclusão}

O estudo teve como objetivo principal calcular um índice de eficiência técnica que permitisse a comparação de cada unidade da Polícia Militar dentro do Estado de Minas Gerais e testar se fatores socioeconômicos, representando características específicas de cada município, afetariam esse índice.

Para tanto, foi utilizada a técnica Análise Envoltória de Dados para obtenção dos escores de eficiência para 851 municípios mineiros, tendo sido observado, de forma geral, que os escores de eficiência se distribuem em torno de uma média igual 0,49 e que a maior concentração dos municípios, 636, localiza-se entre as faixas de eficiência de 0,3 a $0,6 . \mathrm{Na}$ análise, apenas 14 municípios foram tidos como $100 \%$ eficientes da população total, destacando a grande heterogeneidade existente entre eles, além do baixo nível da eficiência média calculada. Esses 14 municípios, ou até mesmo municípios próximos à fronteira de eficiência, como destacado pela a abordagem DEA, poderiam ser utilizados como benckmarks para outros municípios ineficientes, no sentido de tentar elevar seus escores de eficiência.

O teste de hipótese, a respeito dos determinantes dos escores de eficiência 
calculados, rejeitou a hipótese de que tais escores seriam influenciados pelo ambiente socioeconômico encontrado em cada município. Verificou-se que variáveis normalmente relacionadas com altas taxas de criminalidade, como densidade demográfica, percentual da população urbana, percentual de domicílios sem banheiro e água encanada, percentual de mulheres chefe de família, percentual de adolescentes fora da escola em 1991 e tamanho dos municípios, mostraram-se estatisticamente significantes; porém, conjuntamente, todas as variáveis utilizadas explicam apenas $10 \%$ da variabilidade dos escores de eficiência calculados.

Assim, pôde-se concluir que os escores de eficiência calculados não são afetados pelo ambiente socioeconômico particular a cada município e que tais diferenças poderiam representar reais diferenças no nível de eficiência técnica da Polícia Militar de cada unidade. Todavia, esta última hipótese não pôde ser testada, já que não existem dados disponíveis para testar essa pressuposição.

Neste sentido, cabe destacar novamente que o trabalho se limitou a testar apenas a influência socioeconômica dos escores de eficiência, e estudos futuros que incorporem outros insumos utilizados pela polícia, na sua função de policiamento ostensivo, poderão fornecer indicadores de eficiência mais precisos e condizentes com a realidade. Além disso, a obtenção dessas variáveis, diretamente ligadas ao exercício da função, também permitirá testar a hipótese de que tais indicadores sejam realmente representantes dos reais níveis de eficiência de cada unidade da Polícia Militar.

Por fim, mesmo com as limitaçóes impostas, se entende que o estudo contribui com a discussão acerca do combate à criminalidade ao fornecer evidências da importância da análise de eficiência do sistema de segurança pública como um todo. A utilização dos escores de eficiência para identificação das unidades benchmarks pode fornecer indícios para a realização de estudos mais aprofundados nas unidades mais eficientes com o objetivo de identificar quais fatores as diferenciam das outras, permitindo, assim, a construção de políticas públicas de segurança mais eficazes, embasadas em observações empíricas de municípios que adotam métodos ou estratégias de gestâo de seus recursos mais eficientemente do que outros. 


\section{Referências bibliográficas}

ALMEIDA, E. S.; HADDAD; Hewings. The spatial pattern of crime in Minas Gerais: An exploratory analysis. Revista Economia Aplicada, 2005.

ARAUJO JUNIOR, A. F.; FAJNZYLBER, P. Crime e economia: Um estudo das microrregiōes mineiras. Revista Econômica do Nordeste, Fortaleza - CE, v. 31, n. especial, p. 630-659, 2000.

BANKER, R. D.; CHARNES, H.; COOPER, W. W. Some models for estimating technical and scale inefficiencies in data envelopment analysis. Management Science, v. 30, n. 9 , p. 1078-1092, 1984.

\section{BATITUCCI, E. C.;}

RIBEIRO, L. M. L.; CRUZ, M. V. G. Políticas Públicas de Segurança: O caso de Minas Gerais. In: CONGRESSO DA ASSOCIAÇÃO NACIONAL DOS PROGRAMAS DE PÓS-GRADUAÇĀO EM ADMINISTRAÇÃO, 27., 2003, Atibaia/SP. Resumos dos trabalhos apresentados... ENANPAD 2003, 2003.

BEATO FILHO, C. C.; ASSUNCAO, R.; SANTOS, M. A. C.; SANTO, L. E. E.;
SAPORI, L. F.; BATITUCCI, E. Evoluçáo da criminalidade violenta em Minas Gerais 1985-1997. In: REUNIÃO DA ANPOCS, 22., 1997.

Caxambu. Anais... Sessão Violência Justiça e Direitos da XXII Reuniấo da ANPOCS. São Paulo: ANPOCS, 1998.

BEATO FILHO, C. C.

Ação e estratégia das

organizaçóes policiais. In: CONGRESSO DO PROJETO "POLÍCIA E SOCIEDADE DEMOCRÁTICA”, 2., 1999,

Rio de Janeiro. Anais... Polícia e Sociedade Democrática, 1999a.

BEATO FILHO, C. C. Políticas Públicas de Segurança e a questão policial: Eficiência, equidade e accountability. In: MELO,

Marcus André (Org.). Reforma do Estado e Mudança Institucional no Brasil. Recife: Fundação Joaquim Nabuco e Editora Massangano, 1999b, v. 1, p. 335-365.

BEATO FILHO; C. C.

Determinantes da criminalidade em Minas Gerais. Revista Brasileira de Ciências Sociais, v. 13, n. 37, p. 74-87, jun. 1998.

BECKER, G. S. Crime and punishment: An economic approach. Journal of Political Economy, n. 76, p. 169-217, 1968.

CARRINGTON R.; PUTHUCHEARY, N.; ROSE, D. Performance measurement in government service provision: The case of police services in New South Wales. Journal of Productivity Analysis, v. 8, p. 415-430, 1997.

CHARNES, A.; COOPER, W. W.; LEWIN, A. Y.; SEIFORD, L. M. Data envelopment analysis: Theory, methodology, and application. Dordrecht: Kluwer Academic, 1994. 513p.

CHARNES, A.; COOPER, W. W.; RHODES, E. Measuring the efficiency of decision making units. European Journal of Operational Research, v. 2, n. 6, p. 429-444, 1978.

COHEN, Lawrence; FELSON, Marcus. Social change and crime rate trends: A routine approach. American Sociological Review, v. 44, p. 588-608, Aug. 1979. COOPER, W. W.; SEIFORD, L. M.; TONE, K. Data envelopment analysis: A comprehensive text with models, applications, references and $D E A$-Solver software. Norwell, Massachusetts: Kluwer Academic Publishers, 2000. 318p.
FERREIRA, M. A. M.; BRAGA, M. J. Eficiência das sociedades cooperativas e de capital na indústria de laticínios. Revista Brasileira de Economia, v. 61, p. 231-244, 2007

GLAESER, E.; SACERDOTE, B.; SCHEINKMAN, J.

Crime and social interactions. Quarterly Journal of Economics, v. 111, p. 507-548, 1996.

GOMES, A. P.; BAPTISTA, A. J. M. S. Análise envoltória de dados: Conceitos e modelos básicos. In: SANTOS, M. L.; VIEIRA, W. C. (Eds). Métodos quantitativos em economia. Viçosa: UFV, 2004. p. 121-160.

GREENE, W. H. Econometric Analysis. Prentice Hall. 6. ed., 2008. 1216p.

KIRJAVAINEN, Tanja; LOIKKANEN, Heikki A. Efficiency differences of finnish senior secondary schools: An application of DEA and Tobit analysis. Economics of Education Review, v. 17, n. 4, p. 377-394, 1998.

LEVITT, S. D.; DUBNER, S. J. Freakonomics: O lado oculto e inesperado de tudo que nos afeta. Rio de Janeiro: Elsevier, 2005. 
LINS, M. P. E.; MEZA, L. A. Análise envoltória de dados e perspectivas de integração no ambiente de apoio à tomada de decisäo. Rio de Janeiro: COPPE/UFRJ, 2000. 232p.

MARINHO, A.;

FAÇANHA, L. O. Hospitais universitários: Avaliação comparativa de eficiência técnica. Economia Aplicada, v. 4, n. 2, p. 315-349, 2000.

MARINHO, A. Avaliação da eficiência técnica nos serviços de saúde nos municípios do Estado do Rio de Janeiro. Revista Brasileira de Economia, v. 57, n. 2, p. 515-534, 2003.

MARSHALL, R. M. Mapping disease and mortality rates using Empirical Bayes Estimators. In: Journal of the Royal Statistical Society, Series C (Applied Statistics), v. 40, n. 2, p. 283-294, 1991.

MELLO, J. C. C. B. S.; GOMES, E. G.; ASSIS, A. S.; MORAIS, D. P. Eficiência DEA como medida de desempenho de unidades policiais. Revista Produção Online. Florianópolis, v. 5, n. 3, p. 4-16, 2005.

MELLO, J. M. P.; ZILBERMAN, Eduardo. Does crime affect economic decisions? An empirical investigation of savings in a high crime environment. In: ENCONTRO BRASILEIRO DE ECONOMETRIA, 27., 2005, Natal. Anais... Natal, 2005

MESSNER, S. F.; ANSELIN, L. Spatial analyses of homicide with areal data. In: GOODCHILD, M. F.; JANELLE, D. G. (Eds.). Spatially integrated social science (p. 127-144). Oxford, England: Oxford University Press (2004).

NESP - Núcleo de Estudos em Segurança Pública da Fundaçáo João Pinheiro - FJP. Anuário de Informaçōes Criminais de Minas Gerais. Disponível em: $<$ http://www.fjp.gov.br/produtos/ cees/nesp/download_cc.htm>. Acesso em: 01/07/2008

PEIXOTO, B. T.; MORO, Sueli; ANDRADE, Mônica Viegas. Criminalidade na Região Metropolitana de Belo Horizonte. In: ENCONTRO DA ASSOCIAÇÃO NACIONAL DE PÓS-GRADUAÇÃO E PESQUISA EM AMBIENTE E SOCIEDADE, 2.

2004, Indaiatuba, Anais... Indaiatuba, 2004.

PUEEH, F. How do criminals locate? Crime and spatial dependence in Minas Gerais. Clemont Ferrand, France: Université d'Auvergne, 2004. [20 maio 2004]. Disponível em: <http://www4.fe.uc. pt/30years/paper/68.pdf>. Acesso em: 30/11/2006

RIBEIRO, L. M. L.; CRUZ, M. V. G.; BATITUCCI, Eduardo Cerqueira. Desafios à Gestão Democrática das Políticas de Segurança Pública: Análise das recentes transformaçóes em Minas Gerais. In: ENCONTRO NACIONAL DA ASSOCIAÇÃO DE PÓS GRADUAÇÃO E PESQUISA
EM CIÊNCIAS SOCIAIS, 28. 2004, Caxambu - MG. Anais da $28^{a}$ ANPOCS, 2004.

SCALCO, Paulo R.

Criminalidade violenta em Minas Gerais: Uma proposta de alocação de recursos em segurança pública. 2007. Dissertação (Mestrado em Economia) - Departamento de Economia, Universidade Federal de Viçosa, Viçosa - MG, 2007.

SHAO, B.; LIN, W. Technical efficiency analysis of information technology investments: A twostage empirical investigation. Information \& Management, v. 39, p. 391-401, 2002.

SUN, S. Measuring the relative efficiency of police precincts using data envelopment analysis. Socio-Economic Planning Sciences, v. 36, p. 51-71, 2002.

WAISELFISZ, J. J. Mapa da violência 2010: Anatomia dos homicídios no Brasil. Brasília: Instituto Sangari, RITLA, Ministério da Justiça, 2010.

WILSON, James Q.; KELLING, George. The police and neighborhood safety: Broken windos. Atlantic Monthly, p.29-38, Mar. 1982.

E-mail de contato dos autores
pauloscalco@yahoo.com.br
aimorim2007@yahoo.com.br
apgomes@ufv.br
Artigo recebido em janeiro de 2009;
aprovado em dezembro de 2010.




\section{ANEXO}

\section{TABELA A_Resumo descritivo das variáveis socioeconômicas utilizadas no modelo "Tobit"}

\begin{tabular}{|c|c|c|c|c|c|}
\hline & Média & $\begin{array}{l}\text { Desvio } \\
\text { padrão }\end{array}$ & Máximo & Mínimo & Obs. \\
\hline Densidade demográfica (DD) & 59.07 & 286.20 & 6718.00 & 1.40 & 851 \\
\hline Percentual da população urbana (PU) & 0.626 & 0.206 & 1.000 & 0.118 & 851 \\
\hline Índice de Gini (IG) & 0.555 & 0.049 & 0.730 & 0.440 & 851 \\
\hline Taxa bruta de frequência à escola (TBFE) & 0.818 & 0.082 & 0.954 & 0.539 & 851 \\
\hline Percentual de mulheres chefes de família (PMCF) & 0.340 & 0.123 & 0.782 & 0.124 & 851 \\
\hline Renda per capita (RP) & 177.98 & 72.88 & 557.44 & 49.12 & 851 \\
\hline Percentual de domicílios sem banheiro e água encanada (PDSBA) & 0.013 & 0.007 & 0.089 & 0.010 & 851 \\
\hline Percentual de adolescentes fora da escola em 1991 (PAFE) & 0.634 & 0.102 & 0.899 & 0.274 & 851 \\
\hline Percentual de crianças fora da escola em 1991 (PCFE) & 0.229 & 0.078 & 0.557 & 0.589 & 851 \\
\hline
\end{tabular}

Fonte: Atlas de Desenvolvimento Humano - FJP 